\title{
Cross and co resistance among Danish porcine E. coli isolates
}

Jensen, Lars Bogø; Birk, Tina; Borck Høg, Birgitte; Larsen, Lars Stehr; Aabo, Søren; Korsgaard, Helle Bisgaard

\section{Published in:}

Research in Veterinary Science

Link to article, DOI:

10.1016/j.rvsc.2018.07.002

Publication date:

2018

Document Version

Peer reviewed version

Link back to DTU Orbit

Citation (APA):

Jensen, L. B., Birk, T., Borck Høg, B., Larsen, L. S., Aabo, S., \& Korsgaard, H. B. (2018). Cross and co resistance among Danish porcine E. coli isolates. Research in Veterinary Science, 119, 247-249.

https://doi.org/10.1016/j.rvsc.2018.07.002

\section{General rights}

Copyright and moral rights for the publications made accessible in the public portal are retained by the authors and/or other copyright owners and it is a condition of accessing publications that users recognise and abide by the legal requirements associated with these rights.

- Users may download and print one copy of any publication from the public portal for the purpose of private study or research.

- You may not further distribute the material or use it for any profit-making activity or commercial gain

- You may freely distribute the URL identifying the publication in the public portal

If you believe that this document breaches copyright please contact us providing details, and we will remove access to the work immediately and investigate your claim 


\section{Accepted Manuscript}

Cross and co resistance among Danish porcine E. coli isolates

L.B. Jensen, T. Birk, B. Borck Høg, L. Stehr, S. Aabo, H. Korsgaard

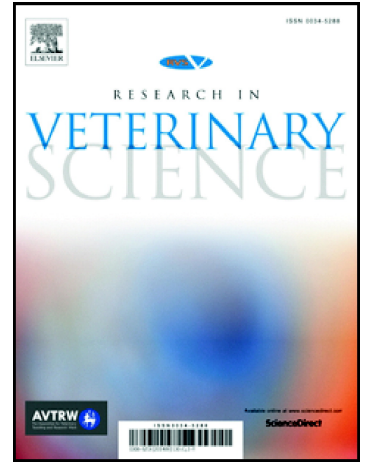

PII:

S0034-5288(18)30131-0

DOI:

doi:10.1016/j.rvsc.2018.07.002

Reference:

YRVSC 3606

To appear in:

Research in Veterinary Science

Received date:

12 February 2018

Revised date:

6 July 2018

Accepted date:

9 July 2018

Please cite this article as: L.B. Jensen, T. Birk, B. Borck Høg, L. Stehr, S. Aabo, H. Korsgaard, Cross and co resistance among Danish porcine E. coli isolates. Yrvsc (2018), doi:10.1016/j.rvsc.2018.07.002

This is a PDF file of an unedited manuscript that has been accepted for publication. As a service to our customers we are providing this early version of the manuscript. The manuscript will undergo copyediting, typesetting, and review of the resulting proof before it is published in its final form. Please note that during the production process errors may be discovered which could affect the content, and all legal disclaimers that apply to the journal pertain. 
Cross and co resistance among Danish porcine E. coli isolates

Jensen, L.B. ${ }^{* 1}$, Birk, T. ${ }^{1}$, Borck Høg, B. ${ }^{1}$, Stehr, L. ${ }^{1}$, Aabo, S. ${ }^{1}$ and Korsgaard, H. ${ }^{1}$

National Food Institute, Technical University of Denmark, Kemitorvet, DK-2800 Kgs. Lyngby,

Denmark

${ }^{*}$ Corresponding author, e-mail: Iboj@food.dtu.dk; phone (+45) 22768169 


\section{Abstract}

Cross and co-resistance to antimicrobials are presented for 765 Danish Escherichia coli isolates of porcine origin from 2009-2013. All isolates and data originate from the DANMAP surveillance but have not previously been used to describe the occurrence of cross and co- resistance. Data presented here clearly indicate the ability of low classified antimicrobials as ampicillin to uphold resistance to critical important antimicrobials for human treatment.

\section{Keywords}

Co resistance, cross resistance, multi resistance, E. coli, ampicillin 
It is generally believed that prevalence of antimicrobial resistance is a result of usage of antimicrobial (Levy 1997). This usage will select for already existing resistance bacteria enhancing their prevalence. General observations of prevalence of resistance have in contradiction to this shown non linear relationship. Major changes of usage can occur while the prevalence of antimicrobial resistance prevails (Aarestrup 1999). Co - and cross resistance mechanisms are most likely responsible for this observation (Pitout and Laupland 2008). In general, surveillance data is not presented in ways that visualize this (Anonymous 2014) and the European Food safety Authority experts have lately suggested that analysis of surveillance data should include clear structured tables that visualize co - and cross resistance (EFSA 2012).

Here we analyze data for 765 porcine E. coli isolates obtained from $2009-2013$ as part of the Danish Integrated Antimicrobial Resistance Monitoring and Research Programme (DANMAP) (Table 1) to investigate for presence of cross- and co-resistance. Isolates were collected from fecal samples collected at slaughterhouses in Denmark each year. Single isolates representing farms in Denmark were isolated by first directly plaiting on Drigalski agar, then picking yellow colonies, incubating these in BBL CHROM agar Orientation Medium and isolated as red colonies from this media after o.n. incubation at $37^{\circ} \mathrm{C}$. For more details see Anonymous 2014.

Isolates were tested for antimicrobial resistance to 16 compounds, representing eight distinct antimicrobial structural families. The compounds include tetracyclines, $\beta$-lactams, sulphonamides, trimethoprim, aminoglycosides, amphenicols, quinolones and polymyxins (colistin) (Anonymous 2014) representing antimicrobial classes, which WHO has classified according to their importance for treatment of human infections (Collignon et al. 2009). Resistance was defined according to EUCAST epidemiological cut-off values and antimicrobial resistance testing was done as microbroth dilution MIC with Sensititre and inoculation procedures according to CLSI guidelines and European standard (ISO 20776-1:2006)(Anonymous 2014) 
Co-resistance was defined as resistance to different antimicrobial compounds belonging to the same chemical structure group ( $\beta$-lactams, aminoglycosides, amphenicols and quinolones). Coresistance can be encoded by one resistance determinant. Cross-resistance is defined as resistance to two or more compounds belonging to different distinct antimicrobial resistance compound and will be encoded by series of antimicrobial resistance determinants.

Overall $45 \%(n=334)$ of the tested isolates were fully susceptible to all antimicrobials tested and $32 \%(n=245)$ were found multi-resistant using the EFSA definition of resistance to more than five antimicrobial families (EFSA 2012),. Resistance profiles were sustained in all tested years. The most dominant multi-resistant profile was resistance to ampicillin (AMP), streptomycin (STR), sulphonamides (SUL), tetracycline (TET) and trimethoprim (TRI); $\%$ ( $n=48)$ of the isolates had this profile. $27 \%$ of all isolates $(n=205)$ were resistant to $\beta$-lactams. All cephalosporin-resistant (tested with the $3^{\text {rd }}$ generation cephalosporins cefotaxime and ceftiofur) isolates $(n=5)$ had crossresistance to ampicillin due to genetic background. Two percent of the ampicillin $(n=205)$ resistant isolates were cross-resistance to cephalosporins.

Forty-two percent of all isolates $(n=320)$ where resistance to streptomycin. Among aminoglycosideresistant isolates, resistance is encoded by numerous resistance genes (Shaw et al. 1993), all giving different patterns for cross-resistance (Hedges and Shannon 1984). 92\% of the spectinomycin-resistant isolates ( $n=159), 85 \%$ of the neomycin-resistant isolates $(n=34), 46 \%$ of the spectinomycin $(n=147)$ and $9 \%$ of the neomycin $(n=9)$ resistant isolates showed crossresistance to streptomycin. All apramycin-resistant isolates $(n=3)$ and gentamicin-resistant isolates $(n=5)$ were cross-resistant to streptomycin due to genetic background for resistance. This has been reported previously (Hedges and Shannon 1984, Sandvang and Aarestrup 2000).

Less than one percent of all tested isolates were resistant to amphenicols but due to common genetic background for resistance all florfenicol-resistant $E$. coli isolates $(n=4)$ had cross-resistance to chloramphenicol. However, only $12 \%$ of the chloramphenicol-resistant $E$. coli isolates $(\mathrm{n}=34)$ showed cross-resistance to florfenicol. 
Less than one percent of the tested isolates $(n=5)$ were quinolone-resistant but due to a shared genetic background for antimicrobial resistance, cross-resistance was observed between resistance to nalidixic acid and ciprofloxacin $(n=5)$.

The highest levels of co-resistance were found among isolates resistant to sulphonamides (five distinct structural groups). Among those isolates $100 \%$ of the quinolone-resistant isolates $(n=5)$, $91-100 \%$ of the amphenicols (chloramphenicol $(91 \%, n=34)$ and florfenicol $(100 \%, n=4)$ ) resistant isolates, $91-100 \%$ of amphenicols (91\% chloramphenicol $(n=34)$ and $100 \%$ florfenicol $(n=4))$ resistant isolates, $93 \%$ of trimethoprim $(n=170)$ resistant isolates, $80-91 \%$ of the aminoglycoside (gentamicin $(80 \%, n=5)$ and neomycin $(91 \%, n=34)$ ) resistant isolates and $80-85 \%$ of the $\beta$ lactams (ampicillin (85\%, $\mathrm{n}=205)$ and 3rd generation cephalosporin $(80 \%, \mathrm{n}=5))$ resistant isolates were co-resistant to sulphonamides.

High co-resistance was furthermore found among isolates resistant to streptomycin (four families), tetracycline (two families), ampicillin (two families) and trimethoprim (one family).

Surprisingly, results presented here shows that while $93 \%$ of the sulphonamides-resistant isolates were co-resistant to trimethoprim only $63 \%$ of trimethoprim resistant isolates were co-resistant to sulphonamides. These two antimicrobials are often used in combination (Anonymous 2014), and therefore, an equal prevalence of co-resistance was expected and even though encoded by difference resistance genes are often found together on plasmids (Freitag et al. 2017). No explanation for this can be given.

Co- and cross-resistance could cause the observed disproportions between the prevalence of resistance and the quantitative usage of some of the antibiotics (Anonymous 2014). Co- and crossresistance to commonly used antimicrobials, such as tetracycline, ampicillin, sulphonamides and streptomycin, may thus uphold resistance to other chemically distinct antimicrobial families. In accordance to this, usage of antimicrobials not listed as critically important for human treatment can uphold already developed resistance to critically important antimicrobials, as well as selecting multi-resistant profiles otherwise limiting treatment possibilities. Co-resistance can thus be the driver for presence of resistance to the critical important antimicrobials for human treatment in $E$. 
coli in Danish pigs several years after the industry has stopped using these antimicrobials, making it extremely difficult to eliminate prevalence of unwanted antimicrobial resistance. Surprisingly, all isolates resistant to $3^{\text {rd }}$ generation cephalosporins, fluoroquinolones and gentamicin were resistant to ampicillin. Cross-resistance between ampicillin and $3^{\text {rd }}$ generation cephalosporins is not surprising, but co-resistance to quinolones and the aminoglycosides apramycin/gentamicin was not expected. This result could indicate that the usage of ampicillin can uphold already acquired resistance to these critical important antimicrobials. The same level of co-and cross resistance between tetracycline and other critical antibiotics was not observed indicating that usage of ampicillin potentially could be more important for upholding critical resistance in pig production in Denmark than tetracycline (Anonymous 2014). Most of the isolates containing resistance to important antimicrobials are multi-resistant leaving few options to eliminate infections. It was also observed that isolates with specific multi-resistant profiles persisted over the study period and made up a fair proportion (32\%) of the 765 randomly picked E.coli isolates from pigs. Distinct resistance profiles, such as AMP-STR-SUL-TET-TRI $(6 \%, n=9)$ prevailed during the whole period. A closer review of all resistance profiles reveals a backbone profile (AMP-STR-SUL) found in $20 \%$ of the isolates (151 of 765 isolates). This combination is found in almost all multi-resistant isolates showing the addition of new antimicrobial families to this backbone.

The data presented shows the presence of cross- and co-resistance among Danish porcine E. coli. Such data have not previously been retrieved from the DANMAP. Data presented here clearly indicate the ability of low-classified antimicrobials as ampicillin to uphold resistance to critical important antimicrobials. 


\section{Reference list}

Aarestrup FM. 1999. Association between the consumption of antimicrobial agents in animal husbandry and the occurrence of resistant bacteria among food animals. Int $\mathrm{J}$ Antimicrob Agents 12:279-85.

Anonymous. Danmap 2014. Use of antimicrobial agents and occurrence of antimicrobial resistance in bacteria from food animals, food and humans in Denmark. ISSN 1600 - 2032. 2014:110.

Collignon P, Powers JH, Chiller TM, Aidara-Kane A, Aarestrup FM. 2009. World Health Organization ranking of antimicrobials according to their importance in human medicine: A critical step for developing risk management strategies for the use of antimicrobials in food production animals. Clin Infect Dis 49:132-41.

European food safety authority. Scientific Report of EFSA. Parma, Italy, 2012.

Freitag,C., Michael, G.B., Kadlec, K., Hassel, M., Schwartz S. 2017. Detection og Plasmidborne exended-spectrum $\beta$-lactamase (ESBL) genes in Escherichia coli isolates from bovine mastitis. Vet. Micro. 200:151-156

Hedges RW, Shannon KP. 1984. Resistance to apramycin in Escherichia coli isolated from animals: detection of a novel aminoglycoside-modifying enzyme. J Gen Microbiol 130:473-82.

Pitout JD and Laupland KB. 2008. Extended-spectrum beta-lactamase-producing Enterobacteriaceae: an emerging public-health concern. Lancet Infect Dis 8: 159-66.

Sandvang D, Aarestrup FM. 2000. Characterization of aminoglycoside resistance genes and class 1 integrons in porcine and bovine gentamicin-resistant Escherichia coli. Microb Drug Resist 6:19-27.

Shaw KJ, Rather PN, Hare RS, Miller GH.1993. Molecular Genetics of Aminoglycoside Resistance Genes and Familial Relationships of the Aminoglycoside-Modifying Enzymes. Microbiol Rev 57:138-63. 
Table 1. Co- and cross-resistance in E. coli isolates from 2009-2013 in pigs.

\begin{tabular}{|c|c|c|c|c|c|c|c|c|c|c|c|c|c|c|c|c|c|c|}
\hline \multirow[b]{2}{*}{$n$} & \multirow[b]{2}{*}{$\begin{array}{c}\mathrm{n} / \mathrm{N}^{*} \\
\%\end{array}$} & \multirow{2}{*}{$\begin{array}{l}\text { Group } \\
\text { ANTIB }\end{array}$} & \multirow{2}{*}{ 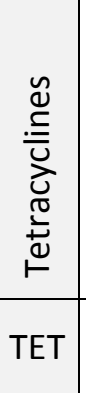 } & \multicolumn{3}{|c|}{$\begin{array}{l}\frac{n}{E} \\
\frac{\pi}{0} \\
\frac{\pi}{1} \\
\infty\end{array}$} & \multirow{2}{*}{ 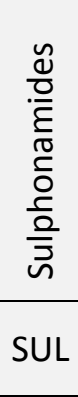 } & \multirow{2}{*}{ 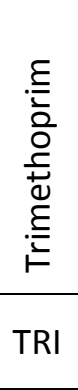 } & \multicolumn{5}{|c|}{ 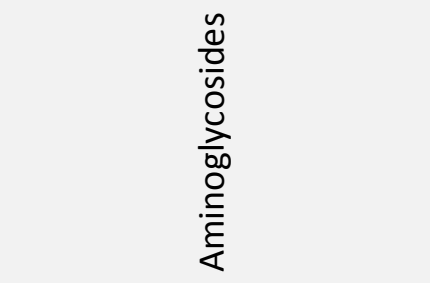 } & \multicolumn{2}{|c|}{$\begin{array}{l}\frac{n}{0} \\
\frac{0}{\frac{0}{d}} \\
\frac{0}{0} \\
\frac{\varepsilon}{\alpha}\end{array}$} & \multicolumn{2}{|c|}{ 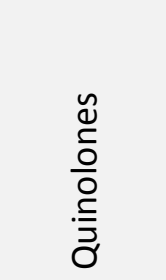 } & \multirow{2}{*}{ 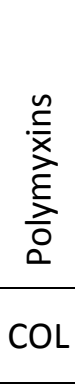 } \\
\hline & & & & AMP & CTX & CEF & & & STR & SPE & NEO & APR & GEN & $\mathrm{CHL}$ & FLO & NAL & CIP & \\
\hline 263 & 34 & TET & 100 & 50 & 1 & 1 & 63 & 44 & 71 & 29 & 10 & 1 & 2 & 8 & 1 & 2 & 2 & \\
\hline 205 & 27 & AMP & 64 & 100 & 2 & 2 & 85 & 64 & 83 & 21 & 11 & 1 & 2 & 9 & 1 & 2 & 2 & \\
\hline 5 & 1 & CTX & 40 & 100 & 100 & 100 & 80 & 60 & 60 & 40 & & n & 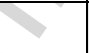 & 20 & & & & \\
\hline 5 & 1 & CEF & 40 & 100 & 100 & 100 & 80 & 60 & 60 & 40 & & 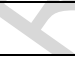 & & 20 & & & & \\
\hline 252 & 33 & SUL & 66 & 69 & 2 & 2 & 100 & 63 & 85 & 33 & 12 & 1 & 2 & 12 & 2 & 2 & 2 & \\
\hline 170 & 22 & TRI & 69 & 78 & 2 & 2 & 93 & 100 & 82 & 28 & 11 & 1 & 2 & 11 & 1 & 2 & 2 & \\
\hline 320 & 42 & STR & 58 & 53 & 1 & 1 & 67 & 43 & 100 & 46 & 9 & 1 & 2 & 7 & 1 & 1 & 1 & \\
\hline 159 & 21 & SPE & 47 & 28 & 1 & 1 & 53 & 30 & 92 & 100 & 8 & 1 & 1 & 11 & 1 & & & \\
\hline 34 & 4 & NEO & 76 & 68 & & & 91 & 53 & 85 & 38 & 100 & & & 3 & & & & \\
\hline 3 & 0.4 & APR & 100 & 100 & & & 67 & 67 & 100 & 33 & & 100 & 100 & 33 & & 33 & 33 & \\
\hline 5 & 1 & GEN & 80 & 100 & & & 80 & 80 & 100 & 20 & & 60 & 100 & 60 & 40 & 20 & 20 & \\
\hline 34 & 4 & $\mathrm{CHL}$ & 59 & 56 & 3 & 3 & 91 & 56 & 68 & 53 & 3 & 3 & 9 & 100 & 12 & 3 & 3 & \\
\hline 4 & 1 & FLO & 50 & 75 & & & 100 & 50 & 100 & 25 & & & 50 & 100 & 100 & & & \\
\hline 5 & 1 & NAL & 100 & 100 & & & 100 & 80 & 80 & & & 20 & 20 & 20 & & 100 & 100 & \\
\hline 5 & 1 & CIP & 100 & 100 & & & 100 & 80 & 80 & & & 20 & 20 & 20 & & 100 & 100 & \\
\hline 2 & 0.3 & $\mathrm{COL}$ & & 50 & & 2 & & & & & & & & & & & & 100 \\
\hline
\end{tabular}

${ }^{*} \mathrm{~N}$ : total number of resistant isolates

Table 1

Table of co- and cross resistance among 765 E. coli of porcine origin isolated in Denmark 2009-2013.

Numbers given are in percentage of the total bacteria resistant to antimicrobials given as columns for the individual tested 16 antimicrobials belonging to eight structural families. Total number of resistant are given in column one (n). Percentage of the total amount of tested bacteria are given in column $n / N$, where $N$ is the total number of tested isolates and $\mathrm{n}$ is the number of resistant isolate to the tested antimicrobial. Percentage below $1 \%$ is excluded from the Table. Abbreviations: TET (tetracycline), AMP (ampicillin), CTX (cefotaxime), CEF (ceftiofur), SUL (sulfonamide), TRI (trimethoprim), STR (streptomycin), SPE (spectinomycin), NEO (neomycin), APR (apramycin), GEN (gentamicin), CHL (chloramphenicol), FLO (florfenicol), NAL (nalidixic 
acid), CIP (ciprofloxacin), COL (colistin). Hatched grey areas indicate higher percentage of co- and cross resistance. 
Highlights

- Presenting prevalence of co- and cross-resistance

- Identifying possible co-selection of antimicrobial resistance among tested isolates

- The importance of usage of ampicillin for production animals 